
FILTRATION (HGMF-TRANSVERSAL) CELL WITH BOUNDED FLOW FIELD

\author{
O. ROTARIU, N. REZLESCU, V. MURARIU AND V. BĂDESCU
}

Magnetic Separation Laboratory, Technical Physics Institute

47 Bd. Mangeron, 6600 laşi, România

(Received April 18, 1997, accepted June 3, 1997)

\begin{abstract}
In this paper we discuss some numerical and experimental results obtained for a magnetic filtration cell with bounded flow field which works in the HGMF-transversal configuration. The numerical results have been obtained by analyzing the particle trajectories in very diluted suspensions, for which the inertia of the particles and the magnetic and hydrodynamic interactions between particles were neglected. The experimental data were obtained by using suspensions of fine particles with magnetic susceptibilities around $5 \cdot 10^{-4} u S I$. Here a comparison between the two categories of results is made for a large range of constructive and operational parameters.
\end{abstract}

\title{
INTRODUCTION
}

In view of obtaining an antierosive and anticorrosive protection of the ferromagnetic matrix and of increasing the filtration efficiency, we designed and analyzed theoretically a new HGMF-transversal magnetic filtration system. It consisted in a set of cells within which the suspension flow fields were bounded 
by pairs of planar parallel walls, behind of which cylindrical ferromagnetic wires were installed in view of producing the magnetic field gradient $[1,2,3]$. By relying on approximation of completely established laminar flow between each cell's parallel walls, we proved that a $100 \%$ filtration efficiency may be obtained with the analyzed cell for adequate constructive and operational parameters.

In this paper we present both some experimental and numerical results regarding efficiency of a single filtration cell and compare them to one another. The numerical data has been obtained by means of a computer program built by us, which calculate the particles' trajectories in a highly diluted suspension and which find the critical trajectories of magnetic capture of the cell ferromagnetic wires. The simulation of the process can furnish the direct calculus of the filtration efficiency by considering the extreme critical trajectories and the particle flux distribution in the flow field.

In view of obtaining the experimental results we have built a filtration cell in which the number of ferromagnetic wires and their spatial distribution may be modified. The experiments were performed with highly diluted suspensions in water, the solid particle sizes laying in the range $50 \div 53 \mu \mathrm{m}$. The operational parameters have been established in same range as those employed in numerical simulation.

\section{THE NUMERICAL SIMULATION}

The filtration cell designed and analyzed by us is sketched in Fig.1. Two planar parallel plates bound the flow field of a uniform suspension of solid paramagnetic particle with radius $b$ and magnetic susceptibility $\chi_{p}$ carried by a fluid of viscosity $\eta_{f}$ and of susceptibility $\chi_{f}$. The flow is directed towards the 


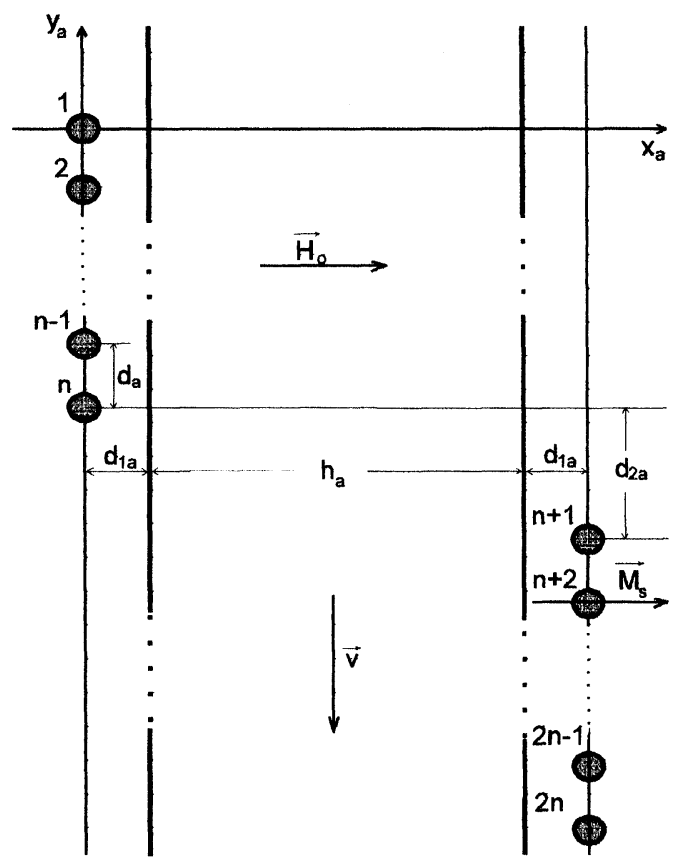

Fig.1 Schematic diagram of the analysed system.

negative direction of the $O y_{a}$ axis, and the flow region is limited by the planes $x_{a}=d_{1 a}=d_{1} / a$ and $x_{a}=d_{1 a}+h_{a}, h_{a}=h / a$ denoting the normalized distance between the plates. Outside the flow field, two groups of $n$ cylindrical ferromagnetic wires each are installed on the plates, parallel to one another. A wire is characterized by its saturation magnetization $M_{s}$, its radius a and has a large length by comparison to $h$. The distance between two neighboring wire axis is $d_{a}=d / a$ and the two groups are spaced by $d_{2 a}=d_{2} / a$ along the plates. The wires of the two groups have their axis in the planes $x_{a}=0$, respectively $x_{a}=h_{a}+2 d_{1 a}$ and are perpendicular to the $O y_{a}$ axis. 
In [3] we described in detail the magnetic force field in the magnetically active space, we obtained the particle motion equations in this space and, from these, the trajectory equations. The computer simulations utilized by us in this work allows one to obtain in the same frame the whole set of critical trajectories, for the caption of each wire; these are functions of the initial coordinates at the particle entrance in the magnetically active space. The filtration efficiency has been determined by computing recovery, defined as the ratio between the flux of particles which follow capture trajectories and the total flux,

$$
R(\%)=\left[1-\frac{\int_{x_{a c}^{n}}^{x_{a c}^{2 n} v_{f} d x_{a}}}{v_{0} h_{a}}\right] \cdot 100
$$

Here $x_{a c}^{n}$ and $x_{a c}^{2 n}$ denote, respectively, the critical trajectories initial coordinates for the $n^{\text {th }}$ and the $2 n^{\text {th }}$ wire, $v_{f}$ denotes local flow velocity, which depends on $x_{a}$ and which is given, under the circumstances of smooth boundary wall surfaces, by the Hagen-Poiseuille relation, and $v_{0}$ is the medium flow velocity of the suspension.

The recovery $R$ in a filtration cell depends, via the initial coordinates of the extreme critical trajectories, on the cell constructive parameters $\left(a, d_{1 a}, d_{2 a}, d_{a}, h_{a}, M_{s}\right)$ and on the operational parameters $\left(b, v_{0}, \chi_{p}, \chi_{f}, \eta_{f}, H_{0}\right)$. The majority of the parameters mentioned are included in the well-known "magnetic velocity" $[4,5,6]$, given by, 
$v_{m}=\frac{2 \mu_{0}\left(\chi_{p}-\chi_{f}\right) b^{2} M_{s} H_{0}}{9 \eta_{f} a}$

for $M_{s}<2 H_{0}$

$v_{m}=\frac{4 \mu_{0}\left(\chi_{p}-\chi_{f}\right) b^{2} H_{0}^{2}}{9 \eta_{f} a}$

for $M_{s}>2 H_{0}$

In Fig.2 there are shown three simulated sets of critical trajectories corresponding to three filtration cells which work with the same operational parameters and being characterised by the same constructive parameters, less the distance $h_{a}$ between the plates which limit the flow field. It can be seen that decrease of this parameter leads to a reduction of the spatial domain of the cell transversal section within which the particle trajectories do not yield a capture. With regard at this, we have defined the limit distance $h_{a}^{100}$ as the greatest value taken by $h_{a}$ in view of obtaining a $100 \%$ recovery for the other functional and constructive parameters being fixed. In Fig. 3 the numerically obtained dependencies of the limit distance $h_{a}^{100}$ on the ratio $v_{m} / v_{0}$ and on the constructive parameters $n$ and $d_{a}$ are given. One can observe the decrease of $h_{a}^{100}$, for a fixed value of $v_{m} / v_{0}$, when the distance $d_{a}$ between the ferromagnetic wire increases. Also, one can note the increase of $h_{a}^{100}$ with the increase of the wire number $n$ and the fact that for $2 x n>20$ this increase is insignificant.

In Fig.5 the dependencies obtained by numerical simulation are presented along with the experimentally obtained recovery dependencies on the operational and constructive parameters. The theoretical ones resemble qualitatively to those known in general for the HGMF filtration systems: the recovery decreases with the increase of the flow velocity of the suspension and 


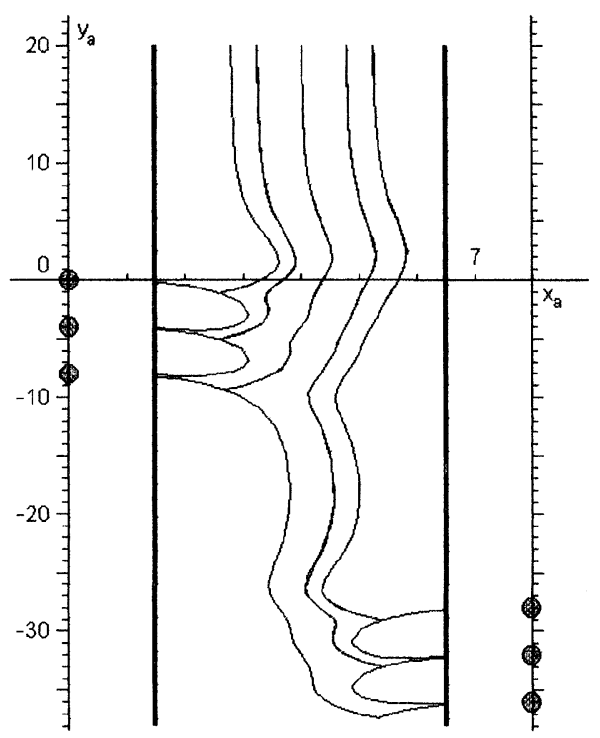

(a)

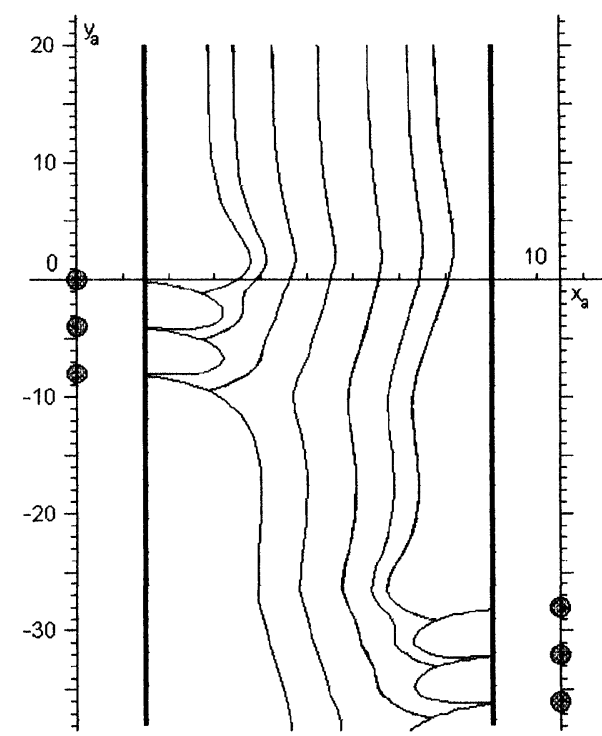

(b)

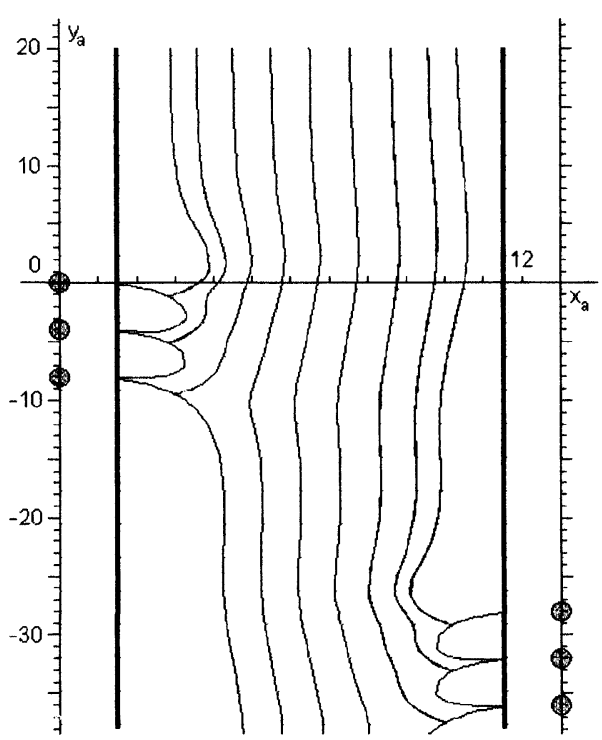

(c)

Fig.2 Critical trajectories for a system having following parameters:

$$
\left.2 \times n=6, d_{a}=4, d_{1 a}=1.5, d_{2 a}=20, v_{m} / v_{0}=30 ; a\right) h_{a}=5 \text {, }
$$$$
R=100 \% \text {; b) } h_{a}=7.5, R<100 \% \text {; c) } h_{a}=10, R<100 \% \text {. }
$$ 
increases with the growth of the applied magnetic field intensity. Particularly, for the filtration cell analysed by us, the recovery grows with the decreases of either the distance between the plates and of that between the ferromagnetic wires. In the following, this behaviour is compared to that established experimentally.

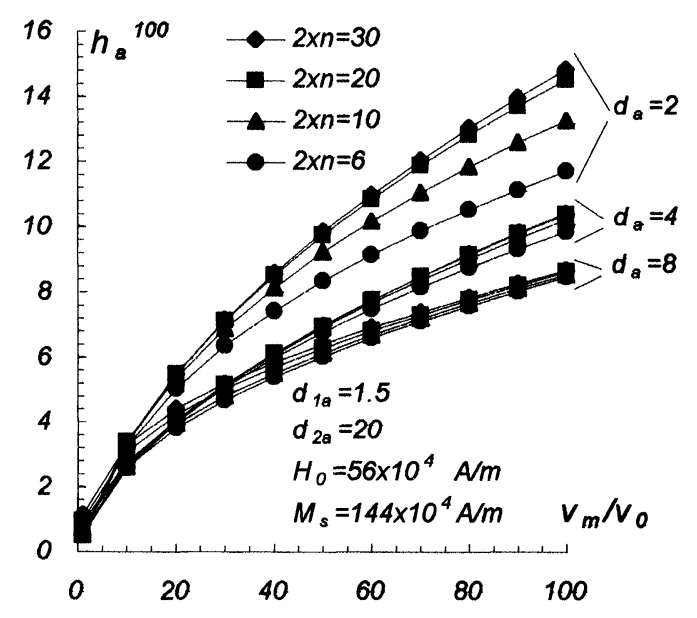

Fig.3 The dependence of the maximum distance $h_{a}^{100}$ separating the planes that restrict the flow for $100 \%$ recovery, on $v_{m} / v_{0}$ ratio.

\section{THE EXPERIMENTAL SET-UP AND THE EXPERIMENTAL DATA}

In the experimental set-up realized by us suspension being filtered flows downwards the vertical of a paralelipipedic box with transparent walls (made of plexiglass) (Fig.4). The main elements of the properly filtration cell are two nonmagnetic plates within which $n$ parallel equidistant slits being cut up. In 
these slits there are mounted the cylindrical ferromagnetic wires of diameters equal to the plate depth and to the slit width. Above the plates which keep the wires, on both sides there are mounted another nonmagnetic plates, much thinner than the former, the last constituting bounds of the flow field between the two assemblies. For our tests we have prepared a lot of wire carrier plates pairs, distinguished by one another by the number of the slits, $n$, and by the distance $d$ between them.

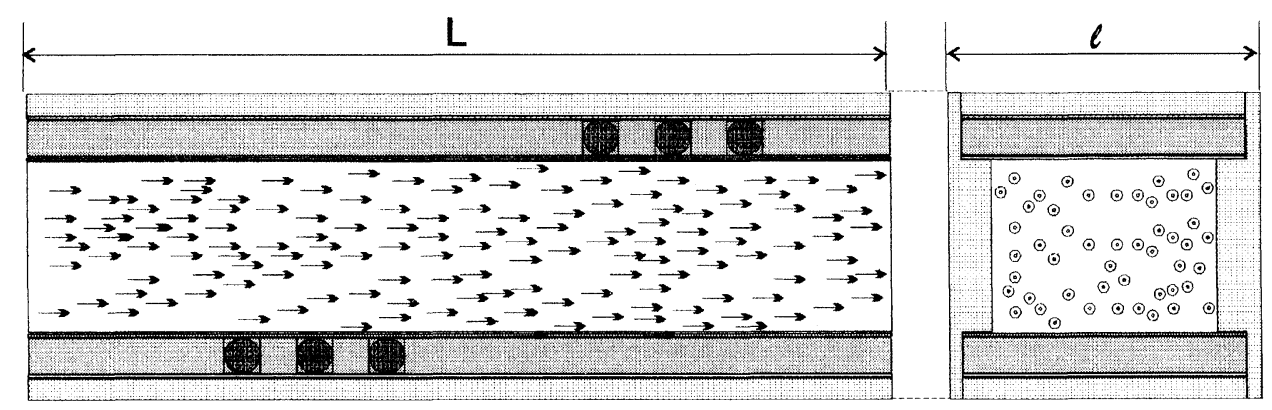

Fig. 4 The experimental set-up for a system with $2 x n=6$ wires (left-longitudinal section; right-transversal section).

The flow channel in our experimental set-up has had a length of $L=500 \mathrm{~mm}$ and a width of $\ell=30 \mathrm{~mm}$, along the wires. In view of reducing the effect of particle deposition at the ends of the ferromagnetic wires, the last has a length larger than the flow channel width. By adding supplementary lateral plates, the width $h$ of the flow field, perpendicularly to the wire axis, can be varied between 3 and $10 \mathrm{~mm}$. The suspension supply of the filtration cell is assured by a recipient with constant fluid level placed above the box, and the medium flow velocity is regulated by means of a tap mounted at the flow channel exit. The carrier liquid in the suspension utilized by us has been water $\left(\eta_{f}=10^{-3} \mathrm{~kg} / \mathrm{m} \cdot \mathrm{s}\right.$ and $\left.\rho_{f}=10^{3} \mathrm{~kg} / \mathrm{m}^{3}\right)$ and the carried solid particles had 
the diameter $2 b=50 \div 53 \mu \mathrm{m}$, the magnetic susceptibility $\chi_{p} \cong 58 \cdot 10^{-4} u S I$ and the density $\rho_{p} \cong 4000 \mathrm{~kg} / \mathrm{m}^{3}$. The volumic fraction of the suspensions laid between $1.5 p p m$ and $30 p p m$. The uniform magnetic field, applied perpendicularly both to the ferromagnetic wires and to the flow direction, was generated by an electromagnet with the pole gap of $60 \times 100 \times 600 \mathrm{~mm}^{3}$ within which the field intensity has been of $H_{0}=4 \cdot 10^{5}-56 \cdot 10^{4} \mathrm{~A} / \mathrm{m}$.

Under the circumstances described above, a large set of tests has been performed, modifying in turn the constructive parameters $(n, d$ and $h)$ and those operational $\left(v_{0}\right.$ and $\left.H_{0}\right)$. For each test the amount of the suspension introduced in the filtration cell was established such that the solid particle mass in the suspension be of $100 \mathrm{mg}$, this representing less than half of the particle mass accumulated for $n=3, h_{a}=10, v_{0}=10 \mathrm{~cm} / \mathrm{s}, H_{0}=4 \cdot 10^{5} \mathrm{~A} / \mathrm{m}$. In order to evaluating the filtration efficiency we have repeated five times each test and we have computed the medium particle mass retained in the filtering cell. By computing the ratio of this mass to that of the total particle mass contained in the circulated suspension in a single test, we have obtained the recovery.

In Fig. 5 there are shown the recovery dependencies on the medium velocity flow of the suspension, for two extreme values of the applied magnetic field intensity and for different constructive parameters of the filtration cell. In the same figure there are shown results of the numerical analysis discussed in the previous section. It can be seen that, for each parameter set, the theoretical results predict the existence of a medium suspension flow velocity for which the recovery is maximum (100\%). The largest recovery obtained experimentally has been of $98 \%$ and it corresponds to the parameters $v_{0}=0.8 \mathrm{~cm} / \mathrm{s}$, $H_{0}=56 \cdot 10^{4} \mathrm{~A} / \mathrm{m}, h_{a}=3.5,2 x n=20, d_{a}=2, d_{1 a}=1.5, d_{2 a}=20$. One can 
also note that, for smaller values of the applied magnetic field intensity $H_{0}$ and for large distances between the planes which bound the flow, a good concordance exists between the computed recovery values and those obtained in experiments.

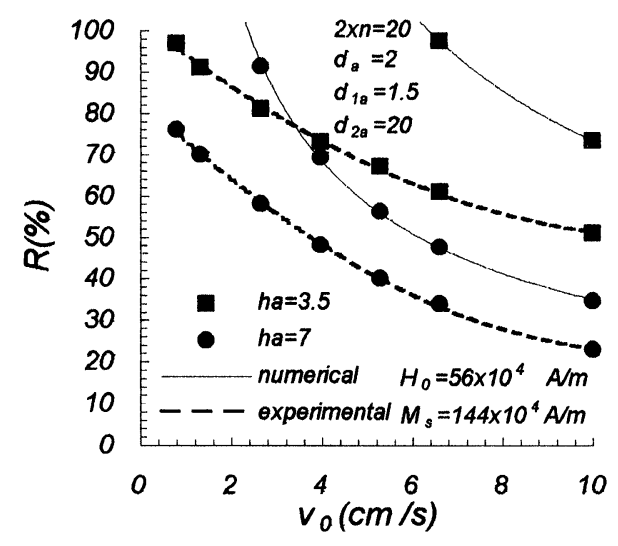

(a)

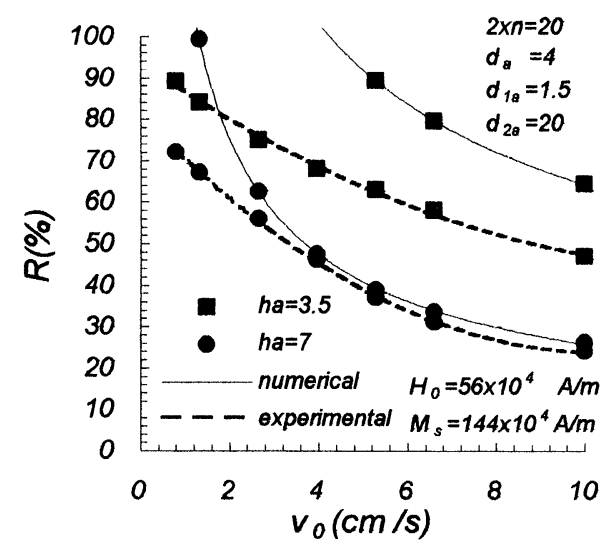

(c)

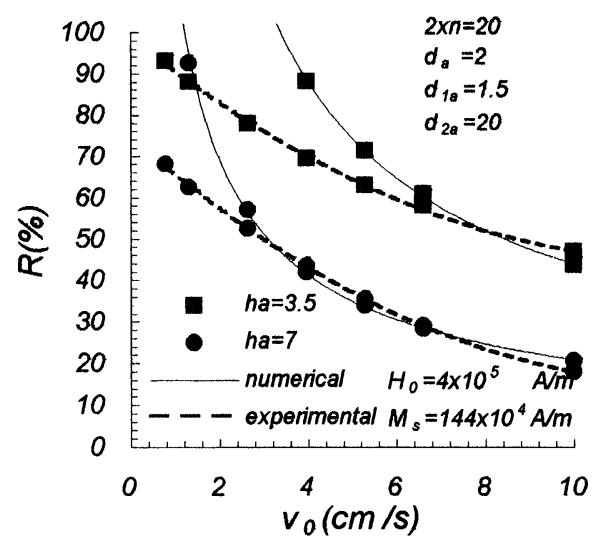

(b)

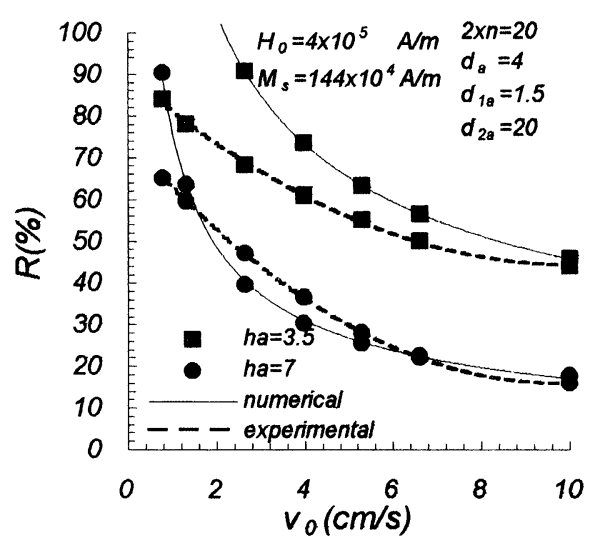

(d)

Fig.5 The recovery dependencies on some operational and constructive parameters. 
The increase of the field intensity and the decrease of either the medium flow velocity or of the distance between the boundary planes yield numerical values of the recovery larger than those obtained experimentally. The difference between them growths when the distance between the wires diminishes. We assume that these disagreements appear as a consequence of ignoring the flowing field perturbation due to the particle deposits which rise on the boundary wall.

\section{CONCLUSIONS}

The possibilities of an efficient filtration by means of the HGMF-transversal magnetic filtration cell with bounded flow field designed and tested by us are revealed by the above described numerical and experimental data. The power of interpretation and of prediction by means of the computer simulations built by us and which involve the suspension particles' trajectory analysis is supported by the qualitative similitude between the numeric and experimental dependencies of the recovery on the constructive and operational parameters. A refinement of these programs should be reached by considering the perturbation of the flow field by the deposits of particles which arise on the walls limiting the flow. Also, an important aspect which should be considered in the force balance is the particle inertia and the volumic fraction of particles in suspension, which is reflected in the hydrodynamic and magnetic interactions between the particle. 


\section{REFERENCES}

1. V. Bădescu, O. Rotariu, V. Murariu and N. Rezlescu, Int. J. Appl. Electromagn. and Mech., Z, 57 (1996).

2. V. Bădescu, O. Rotariu, V. Murariu and N. Rezlescu, Int. J. Multiphase Flow, Vol. 22, 4, 797 (1996).

3. V. Bădescu, O. Rotariu, V. Murariu and N. Rezlescu, IEEE Trans. Magn., in press.

4. J.H.P. Watson, J. Appl. Phys., 44, 4209 (1973).

5. S. Uchiyama, M. Takayasu and S. Kurinobu, Electrical Engineering in Japan, Vol. 97, 4, 459 (1977).

6. R.R. Birss, R. Gerber, M.R. Parker and T.J. Sheerer, IEEE Trans. Magn., 14, 1165 (1978).

\section{ACKNOWLEDGMENT}

The financial contribution from the Ministry of Research and Technology is gratefully acknowledged.

Keywords: Magnetic filtration, critical trajectories, recovery efficiency.

O. Rotariu: for biography, see Magn. Electr. Sep. 8 (1996), 40

N. Rezlescu: for biography see Magn. Electr. Sep. 6 (1995), 151

V. Murariu: for biography see Magn. Electr. Sep. 8 (1996), 40

V. Badescu: for biography see Magn. Electr. Sep. 6 (1995), 151 